\title{
Peripheral Leukocytosis Predicts Cognitive Decline but Not Behavioral Disturbances: A Nationwide Study of Alzheimer's and Parkinson's Disease Patients
}

\author{
Santiago R. Unda ${ }^{\text {a }}$ Aldana M. Antoniazzi $^{b}$ David J. Altschul ${ }^{c, d}$ \\ Roberta Marongiu ${ }^{a}$ \\ aDepartment of Neurological Surgery, Weill Cornell Medicine, Cornell University, New York, NY, USA; \\ ${ }^{b}$ National University of Rosario, Santa Fe, Argentina; 'Department of Neurological Surgery, Montefiore \\ Medical Center, Bronx, NY, USA; ${ }^{\mathrm{d}}$ Albert Einstein College of Medicine, Bronx, NY, USA
}

\section{Keywords}

Parkinson's disease - Alzheimer's disease - Leukocytosis .

Dementia $\cdot$ Behavioral disturbances

\begin{abstract}
Introduction: Peripheral and central nervous system inflammation have been linked to the classic symptoms of Parkinson's disease (PD) and Alzheimer's disease (AD). However, it remains unclear whether the analysis of routine systemic inflammatory markers could represent a useful prediction tool to identify clinical subtypes in patients with Parkinson's and Alzheimer's at higher risk of dementia-associated symptoms, such as behavioral and psychological symptoms of dementia (BPSD). Methods: We performed a multivariate logistic regression using the 2016 and 2017 National Inpatient Sample with International Classification of Diseases 10th edition codes to assess if pro-inflammatory white blood cells (WBCs) anomalies correlate with dementia and BPSD in patients with these disorders. Results: We found that leukocytosis was the most common WBC inflammatory marker identified in 3.9\% of Alzheimer's and 3.3\% Parkinson's patients. Leukocytosis was also found to be an independent risk factor
\end{abstract}

karger@karger.com www.karger.com/dem

Karger $\stackrel{\text { ' }}{5}$

GOPEN ACCESS
(C) 2021 The Author(s)

Published by S. Karger AG, Basel

This is an Open Access article licensed under the Creative Commons Attribution-NonCommercial-4.0 International License (CC BY-NC) (http://www.karger.com/Services/OpenAccessLicense), applicable to the online version of the article only. Usage and distribution for commercial purposes requires written permission. for Parkinson's dementia. Multivariate analysis of both cohorts showed that leukocytosis is significantly decreased in patients with BPSD compared to patients without BPSD. Conclusions: These results suggest a link between leukocytosis and the pathophysiology of cognitive dysfunction in both PD and AD. A better understanding of the role of systemic neuroinflammation on these devastating neurodegenerative disorders may facilitate the development of costeffective blood biomarkers for patient's early diagnosis and more accurate prognosis.

(c) 2021 The Author(s)

Published by S. Karger AG, Basel

\section{Introduction}

Chronic inflammation is a core pathophysiological factor contributing to neurodegenerative diseases [1]. Specifically, previous studies have shown that chronic inflammation may be involved in the early stages of Alzheimer's disease (AD) and Parkinson's disease (PD) [25]. Activation of central nervous system (CNS) resident immune cells produces neuronal death through the release of pro-inflammatory factors, such as cytokines, 
prostaglandins, reactive oxygen species, and other complement proteins [6, 7]. Recent studies have also suggested that peripheral immune cell activation closely contributes to amplify or even trigger CNS immune response in the early stages of neurodegeneration [8-13].

$\mathrm{AD}$ and $\mathrm{PD}$ are the 2 most prevalent neurodegenerative diseases worldwide, which mainly affect the elderly population [14-17]. There are still many open questions regarding the mechanisms that trigger accumulation of pathological toxic protein species leading to neurodegeneration in $\mathrm{AD}$ and $\mathrm{PD}$. Even though these diseases present different core symptoms - cognitive deficits in $\mathrm{AD}$ and motor dysfunction in PD - and unique neuropathology, in both the presence of systemic inflammation is a common denominator which correlates with clinical features $[18,19]$. The prospective Framingham study, a multigenerational, community-based population study, revealed that cognitive-intact patients with higher levels of TNF- $\alpha$ and IL- $1 \beta$ produced by peripheral blood mononuclear cells have nearly 3 -fold higher risk of developing $\mathrm{AD}$ [19]. In PD patients, systemic inflammation is related not only to the onset of motor impairment, but also to its progression. Elevated blood levels of C-reactive protein were associated with early stages of PD [9], freezing of gate [18], and rapid progression of motor phenotype [20].

A wide range of other clinical features associated with the core symptoms of $\mathrm{AD}$ and $\mathrm{PD}$ can occur throughout the disease progression, and they have been related with reduced quality of life, clinical deterioration, and greater mortality risk [21-23]. In $\mathrm{AD}$, up to $40-60 \%$ of patients exhibit behavioral and psychological symptoms of dementia (BPSD) [24], which include mood disorders, sleep disorders, psychotic symptoms, and agitation [25]. Cognitive and associated behavioral symptoms are also common in $\mathrm{PD}$, which may occur even in the prodromal stages of the disease and worsen with its progression [26]. PD patients are 6 times more likely to develop mild cognitive impairment (MCI) or dementia, known as PD dementia (PDD), compared to healthy controls $[27,28]$. Both cognitive impairment and BPSD are a major concern for $\mathrm{AD}$ and $\mathrm{PD}$ patients and their care providers due to its significant humanistic and societal impact $[25,27]$. Patients with dementia and BPSD have substantially greater utilization of healthcare resources, therefore incurring in greater costs and economic expenses [25]. Suitable biomarkers to guide treatment decisions for these associated clinical features are lacking and urgently needed. A few diagnostic tests, such as positron emission tomography scans or cerebrospinal fluid analysis, have been proposed to predict different stages of $\mathrm{AD}$ and $\mathrm{PD}$; yet the cost and invasiveness of these methods represent an important limitation [29]. Thus, blood-based biomarkers that reflect the progression of the underlying neurodegenerative process in these diseases could improve clinical prediction of disease severity and clinical subtypes in large-scale populations [29].

The nervous system is particularly vulnerable to damage in response to systemic inflammation. Cognitive dysfunction and delirium can be induced directly by inflammation-induced infiltration of immune cells and soluble factors such as TNF, IL-1, as well as indirectly through activation of CNS microglia and astrocytes. For example, activation of systemic inflammation by infectious agents has been linked to PD [30] and AD onset [31]; although, the level and chronicity of systemic inflammation required to develop cognitive impairment and BPSD is not fully understood.

We hypothesized that the higher levels of systemic inflammatory markers contribute to $\mathrm{AD}$ and $\mathrm{PD}$ cognitive features compared to healthy controls. A few studies reported systemic inflammatory biomarkers with a potential to identify patients at higher risk of dementia or BPSD in $\mathrm{AD}$ and $\mathrm{PD}$ [32-34]. However, due to the relatively small sample-size populations, it still remains unclear whether the analysis of routine systemic inflammatory markers could represent a useful prediction tool to identify clinical subtypes in patients with $\mathrm{AD}$ and $\mathrm{PD}$ at higher risk of dementia-associated symptoms, such as BPSD. In this study, we aimed to test our hypothesis that elevation in peripheral immune cell levels will correlate with cognitive deficit and BPSD in AD and PD using the largescale National Inpatient Sample (NIS) database.

\section{Material and Methods}

Data Source

Our retrospective cohort study used the 2016 and 2017 NIS database. This large database contains discharges from a 20\% sample of all US hospitals in states participating in the Healthcare Cost and Utilization Project. It contains demographic, diagnostic, and procedural data, with diagnoses and procedures captured in the form of International Classification of Diseases 10th edition (ICD-10).

\section{Study Sample}

Primary inclusion criteria were patients with a discharge diagnosis of MCI or dementia due to AD (ICD-10th: G30) [35, 36] or idiopathic PD (ICD-10th: G20) from January 1, 2016 to December 31,2017 . Secondary inclusion criteria were the presence of neurocognitive disorder with BPSD [37-39] and white blood cells (WBCs) anomalies in AD and PD. Exclusion criteria comprised of patients under 18 years old and a diagnosis of gastrointestinal, urogenital, respiratory infection, bacteremia, viral infection, sepsis, or fever. Two cohorts of age-matched healthy controls to AD or PD cases were also included in the analyses. 


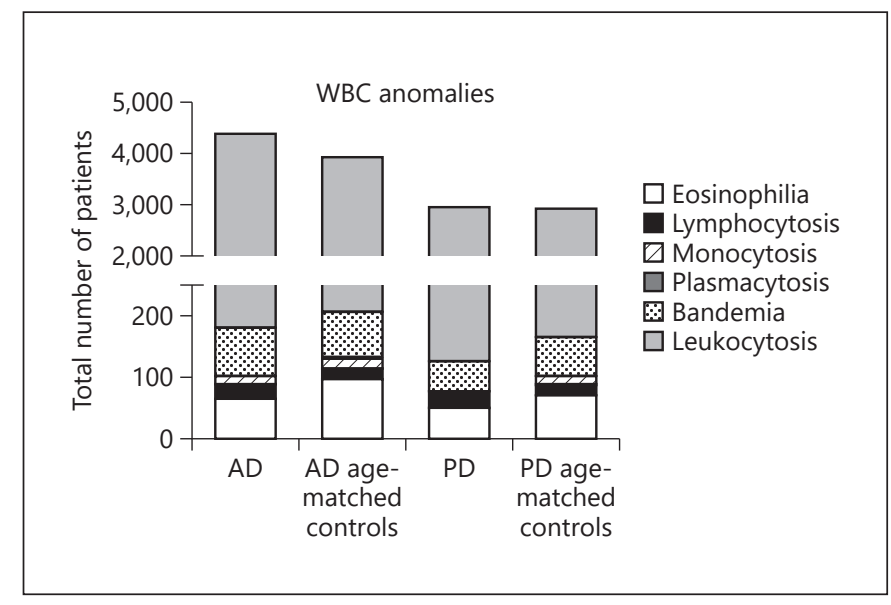

Fig. 1. Leukocytosis is the most prevalent pro-inflammatory WBC anomaly reported in $\mathrm{AD}$ and $\mathrm{PD}$ patients. Graph represents the total number of AD and PD patients registered in the 2016 and 2017 NIS and selected age-matched controls for the study. AD, Alzheimer's disease; PD, Parkinson's disease; WBCs, white blood cells; NIS, National Inpatient Sample.

\section{Collected Data}

The examined demographic data included age, sex, race, and median household income categorized in quartiles. Moreover, long-term (current) treatment with antibiotics was included. The analyzed comorbidities were hypertension, diabetes mellitus with and without complications, lipidemias, congestive heart failure, obesity, depression, history of cerebrovascular disease, current smoker status, and sleep apnea. Chronic pro-inflammatory diseases comprising ulcerative colitis, Crohn's disease, inflammatory polyarthropathies, systemic lupus erythematous, multiple sclerosis, chronic obstructive pulmonary disease, and asthma were also included.

Dementia cases due to disorders which cause mild or major cognitive symptoms were taken into account when performing univariate analysis. These comprised Huntington's disease, frontotemporal dementia, dementia due to head trauma, schizophrenia, vascular dementia, and Lewy body dementia. The most prevalent pro-inflammatory WBC anomalies found in the 2016-2017 NIS database were selected, including eosinophilia, lymphocytosis, monocytosis, plasmacytosis, bandemia, and elevated WBC count (leukocytosis). Specifically, leukocytosis is defined as an elevated WBC count greater than 11,000 per $\mathrm{mm}^{3}\left(11.0 \times 10^{9}\right.$ per L) [40]. All the codes for this study are shown in the online suppl. Table S1; see www.karger.com/doi/10.1159/000516340 for all online suppl. material.

\section{Outcomes}

The primary outcome analyzed for $\mathrm{AD}$ patients was the development of BPSD (ICD-10th: F02.81). For PD patients, the primary outcomes included MCI (ICD-10th: G31.84), major neurocognitive disorder (MND) with or without behavioral disturbances (ICD-10th: F02.80, F02.81), and 2 separate outcomes from the "major neurocognitive disorder" variable, PDD (ICD-10th: F02.80) and PDD with BPSD (ICD-10th: F02.81).

Leukocytosis and Neurocognitive Decline in Alzheimer's and Parkinson's Disease

\section{Statistical Analysis}

Interactions between specific types of pro-inflammatory WBC anomalies and primary outcomes were assessed using the SPSS v.24 software. A univariate analysis was performed to identify unevenly distributed variables in the WBC anomalies that could constitute confounding factors. For this step, all continuous values are represented using mean \pm standard deviation, or median and interquartile range. Categorical variables were described using frequencies and proportions. Comparisons were performed using Student's $t$ test, the nonparametric Mann-Whitney test, or $\chi^{2}$ tests as appropriate.

Factors with a $p$ value $<0.10$ were then included in a multiple logistic regression analysis to reflect the adjusted association between WBC anomalies and each of the primary outcomes. Odds ratios (OR) and 95\% confidence intervals (CIs) were calculated, and a $p$ value $<0.05$ was considered as statistically significant.

In order to test the extent to which pro-inflammatory WBC anomalies could predict the development of neurocognitive alterations and neurodegenerative disease itself, we performed a propensity matched score 1:1 with age-matched healthy controls for each $\mathrm{AD}$ and PD patient. The differences between cases and controls in sociodemographic, comorbidities, and other variables were assessed with a univariate analysis followed by a multiple logistic regression with the same criteria described above.

\section{Results}

A total of 179,102 patients with $\mathrm{AD}$ and 134,501 patients with a diagnosis of idiopathic PD were captured in the 2016 and 2017 NIS database. Out of those, 68,966 patients were excluded from the AD cohort and 43,969 from the PD cohort due to the presence of a diagnosis code of infection or fever. Four thousand four hundred seventeen $\mathrm{AD}$ patients (4\%) and 2,995 PD patients (3.3\%) had at least one of the pro-inflammatory WBC parameters included. The most prevalent anomaly was leukocytosis with 3.9\% $(n=4,244)$ in $\mathrm{AD}$ and $3.2 \%(n=2,870) \mathrm{PD}$ patients. Eosinophilia and bandemia were reported in $0.1 \%$ of patients in both cohorts. The rest of WBC anomalies represented nearly the $0 \%$ of the cases (Fig. 1). WBC anomalies other than leukocytosis were not considered for further analyses due to the low number of cases in our cohorts (online suppl. Tables S2-S6 show the extended values for these analyses).

$\mathrm{AD}$ patients with MCI represented $0.3 \%$ of total cases $(n=312)$, while patients with dementia without BPSD represented $77.5 \%(n=85,418)$ and patients with BPSD $22.2 \%(n=24,405)$ of the entire cohort (Fig. 2a). In the $\mathrm{AD}$ cohort, the analysis between the primary outcomes and WBC anomalies showed that leukocytosis appears as frequent in MCI and dementia cases ( $3.9 \% n=13$ vs. $4.1 \%$ $n=3,510$, n.s.). Interestingly, leukocytosis was significantly more prevalent in Alzheimer's dementia without 

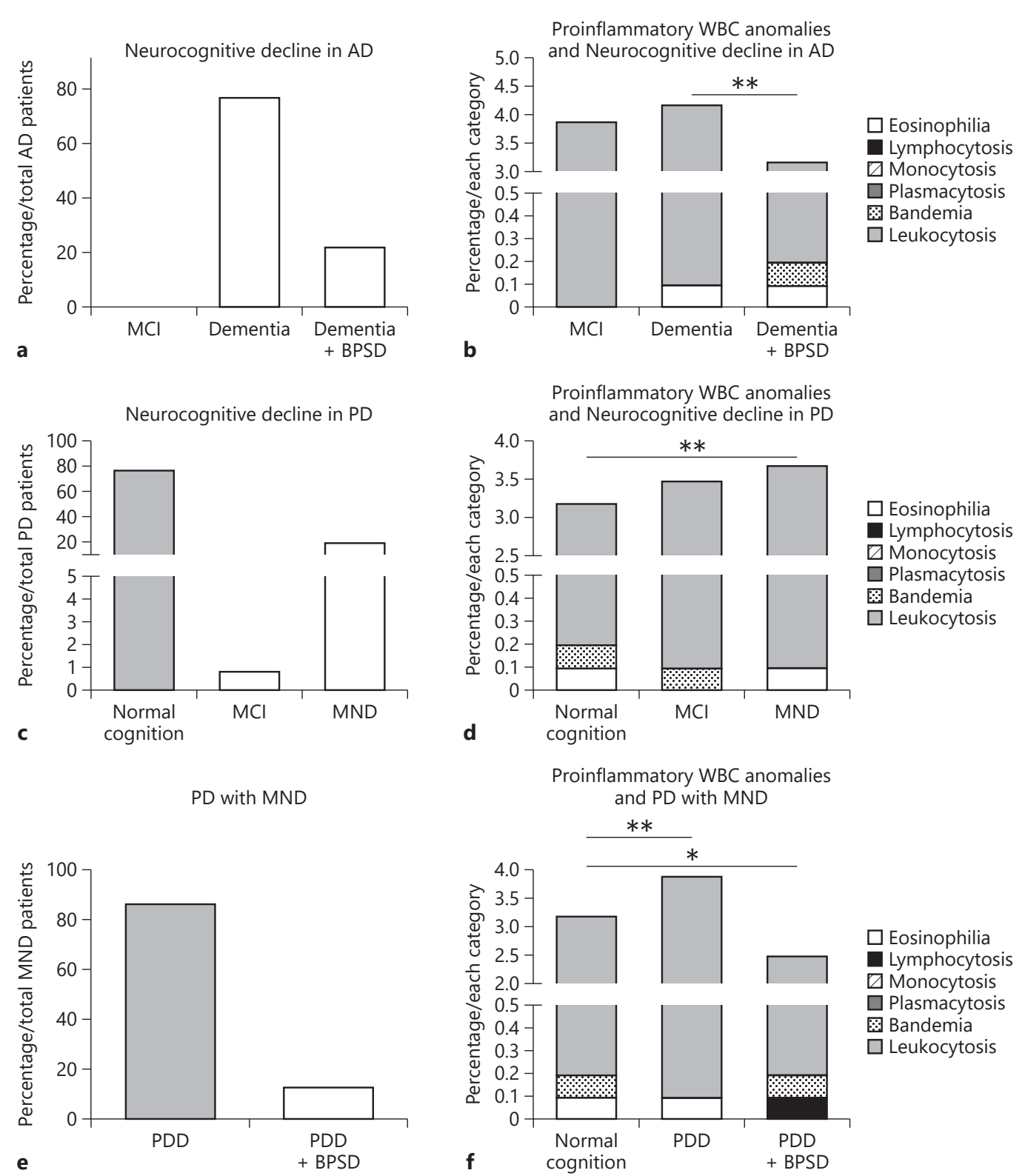

Fig. 2. Higher rates of leukocytosis are found in $A D$ and $P D$ patients with worsen neurocognitive decline. a Rates of MCI, dementia, and dementia + BPSD in AD. b Pro-inflammatory WBC anomalies in $\mathrm{AD}$ patients with MCI, dementia, and dementia + BPSD. c Rates of MCI and MND in PD patients. d Pro-inflammatory WBC anomalies in PD patients with normal cognition, MCI, and MND. e Rates of PDD and PDD + BPSD in PD patients with MND. f Pro-inflammatory WBC anomalies in PD patients with normal cognition, PDD, and PDD + BPSD. Graphs repre- sent the percentage of $\mathrm{AD}$ and $\mathrm{PD}$ patients with $\mathrm{WBC}$ anomalies with respect to the total number of $\mathrm{AD}$ or $\mathrm{PD}$ patients registered in the 2016 and 2017 NIS database. Statistical analysis was carried out using the $\chi^{2}$ test; ${ }^{*} p<0.05,{ }^{* *} p<0.001$. AD, Alzheimer's disease; PD, Parkinson's disease; WBCs, white blood cells; MCI, mild cognitive impairment; MND, major neurocognitive disorder; PDD, Parkinson's disease dementia; BPSD, behavioral and psychological symptoms of dementia; NIS, National Inpatient Sample. 
Table 1. Univariate analysis of AD and PD patients with/without elevated WBCs count (leukocytosis) from the 2016 to $2017 \mathrm{NIS}$ database

\begin{tabular}{|c|c|c|c|c|c|c|}
\hline \multirow[t]{3}{*}{ Variables } & \multicolumn{3}{|l|}{$\mathrm{AD}$} & \multicolumn{3}{|l|}{ PD } \\
\hline & \multicolumn{2}{|l|}{ WBCs count } & \multirow[t]{2}{*}{$p$ value } & \multicolumn{2}{|l|}{ WBCs count } & \multirow[t]{2}{*}{$p$ value } \\
\hline & $\begin{array}{l}\text { normal } \\
(n=105,882)\end{array}$ & $\begin{array}{l}\text { elevated } \\
(n=4,244)\end{array}$ & & $\begin{array}{l}\text { normal } \\
(n=87,658)\end{array}$ & $\begin{array}{l}\text { elevated } \\
(n=2870)\end{array}$ & \\
\hline Age, mean (SD), years & $81.73(7.81)$ & $81.84(7.71)$ & 0.357 & $75.9(9.96)$ & $76.19(9.84)$ & $0.098^{*}$ \\
\hline Female sex, $n(\%)$ & $61,882(58.4)$ & $2,627(61.9)$ & $<0.001^{*}$ & $34,340(39.2)$ & $1,146(39.9)$ & 0.420 \\
\hline \multicolumn{7}{|l|}{ Race, $n(\%)$} \\
\hline White & $76,461(74.6)$ & $3,183(77.2)$ & \multirow[t]{4}{*}{$<0.001^{*}$} & $68,452(80.9)$ & $2,311(83)$ & \multirow[t]{4}{*}{$0.012^{*}$} \\
\hline African-American & $12,056(11.8)$ & $380(9.2)$ & & $5,727(6.8)$ & $151(5.4)$ & \\
\hline Hispanic & $9,037(8.8)$ & $390(9.5)$ & & $6,290(7.4)$ & $176(6.3)$ & \\
\hline Other & $4,943(4.8)$ & $169(4.1)$ & & $4,171(4.9)$ & $147(5.3)$ & \\
\hline \multicolumn{7}{|l|}{ Socioeconomic status, $n(\%)$} \\
\hline Quartile 1 & $30,383(29.1)$ & $1,132(27)$ & \multirow{4}{*}{$0.007^{*}$} & $22,217(25.7)$ & $720(25.4)$ & \multirow{4}{*}{0.624} \\
\hline Quartile 2 & $27.257(26.1)$ & $1,107(26.4)$ & & $22,401(25.9)$ & $747(26.3)$ & \\
\hline Quartile 3 & $24,860(23.8)$ & $1,011(24.1)$ & & $21,575(25)$ & $686(24.2)$ & \\
\hline Quartile 4 & $21,803(20.9)$ & $948(22.6)$ & & $20,186(23.4)$ & $686(24.2)$ & \\
\hline \multicolumn{7}{|l|}{ Comorbidities, $n(\%)$} \\
\hline Hypertension & $69,130(65.3)$ & $2,908(68.5)$ & $<0.001^{*}$ & $53,415(60.9)$ & $1,884(65.6)$ & $<0.001^{*}$ \\
\hline Diabetes mellitus simple & $23,575(22.3)$ & $962(22.7)$ & 0.535 & $20,258(23.1)$ & $719(25.1)$ & $0.015^{*}$ \\
\hline Diabetes mellitus complicated & $10,834(10.2)$ & $381(9)$ & $0.008^{*}$ & $10,278(11.7)$ & $294(10.2)$ & $0.015^{*}$ \\
\hline Lipidemias & $48,984(46.3)$ & $1,978(46.6)$ & 0.656 & $40,419(46.1)$ & $1,279(44.6)$ & 0.103 \\
\hline Obesity & $5,771(5.4)$ & $228(5.4)$ & 0.827 & $8,448(9.6)$ & $281(9.8)$ & 0.783 \\
\hline Congestive heart failure & $25,591(24.2)$ & $853(20.1)$ & $<0.001^{*}$ & $20,535(23.4)$ & $562(19.6)$ & $<0.001^{*}$ \\
\hline Cerebrovascular disease & $13,127(12.4)$ & $433(10.2)$ & $<0.001^{*}$ & $9,664(11)$ & $282(9.8)$ & $0.043^{*}$ \\
\hline Depression & $21,263(20.1)$ & $827(19.5)$ & 0.344 & $17,923(20.4)$ & $578(20.1)$ & 0.689 \\
\hline Current smoker & $5,429(5.1)$ & $253(6)$ & $0.016^{*}$ & $5,713(6.5)$ & $215(7.5)$ & $0.038^{*}$ \\
\hline Sleep apnea & $5,107(4.8)$ & $141(3.3)$ & $<0.001^{*}$ & $8,828(10.1)$ & $252(8.8)$ & $0.024^{*}$ \\
\hline \multicolumn{7}{|l|}{ Other chronic inflammatory diseases, $n(\%)$} \\
\hline Ulcerative colitis & $214(0.2)$ & $16(0.4)$ & $0.014^{*}$ & $280(0.3)$ & $10(0.3)$ & 0.787 \\
\hline Crohn's disease & $237(0.2)$ & $11(0.3)$ & 0.634 & $320(0.4)$ & $14(0.5)$ & 0.286 \\
\hline Inflammatory polyarthropathies & $5,745(5.4)$ & $231(5.4)$ & 0.960 & $5,277(6)$ & $190(6.6)$ & 0.184 \\
\hline Systemic lupus erythematosus & $29(0.03)$ & $0(0)$ & 0.281 & $38(0.04)$ & $0(0)$ & 0.265 \\
\hline Multiple sclerosis & $170(0.2)$ & $8(0.2)$ & 0.657 & $266(0.3)$ & $12(0.4)$ & 0.275 \\
\hline Chronic obstructive pulmonary disease & $13,209(12.5)$ & $528(12.4)$ & 0.949 & $11,230(12.8)$ & $345(12)$ & 0.213 \\
\hline Asthma & $4,393(4.1)$ & $189(4.5)$ & 0.330 & $5,047(5.8)$ & $197(6.9)$ & $0.013^{*}$ \\
\hline \multicolumn{7}{|l|}{ Other causes of dementia, $n(\%)$} \\
\hline $\mathrm{AD}$ & - & - & - & $4,438(5.1)$ & $164(5.7)$ & 0.118 \\
\hline PD & $4,430(4.2)$ & $172(4.1)$ & 0.676 & - & - & - \\
\hline Huntington & $29(0.03)$ & $2(0.05)$ & 0.452 & $27(0.03)$ & $2(0.1)$ & 0.251 \\
\hline Frontotemporal dementia & $224(0.2)$ & $9(0.2)$ & 0.994 & $87(0.1)$ & $5(0.2)$ & 0.215 \\
\hline Dementia due to head trauma & $34(0.03)$ & $2(0.05)$ & 0.596 & $33(0.04)$ & $3(0.1)$ & $0.077^{*}$ \\
\hline Schizophrenia & $1,561(1.5)$ & $63(1.5)$ & 0.957 & $1,995(2.3)$ & $74(2.6)$ & 0.286 \\
\hline Vascular dementia & $4,765(4.5)$ & $152(3.6)$ & $0.005^{*}$ & $1,220(1.4)$ & $30(1)$ & 0.118 \\
\hline Lewy body dementia & $710(0.7)$ & $36(0.8)$ & 0.166 & $641(0.7)$ & $28(1)$ & 0.133 \\
\hline
\end{tabular}

WBCs, white blood cells; AD, Alzheimer's disease; PD, Parkinson's disease; NIS, National Inpatient Sample. * Included in multivariate analysis.

BPSD compared to AD patients with BPSD (3 vs. 4.1\%, $p<0.001$ ) (Fig. 2b; online suppl. Table S2).

In the PD cohort, MCI was present in $0.9 \%(n=707)$ of total cases, while MND was reported for $20.8 \%$ ( $n=$ 18,815) of total PD patients (Fig. 2c). Out of the PD pa- tients with MNDs, $13.3 \%(n=2,499)$ presented BPSD and therefore, $86.7 \%(n=16,316)$ had PDD alone (Fig. 2e). Among the entire PD cohort, we found that leukocytosis prevalence was significantly higher in patients with MND compared to patients presenting normal cognition $(3.6 \%$, 


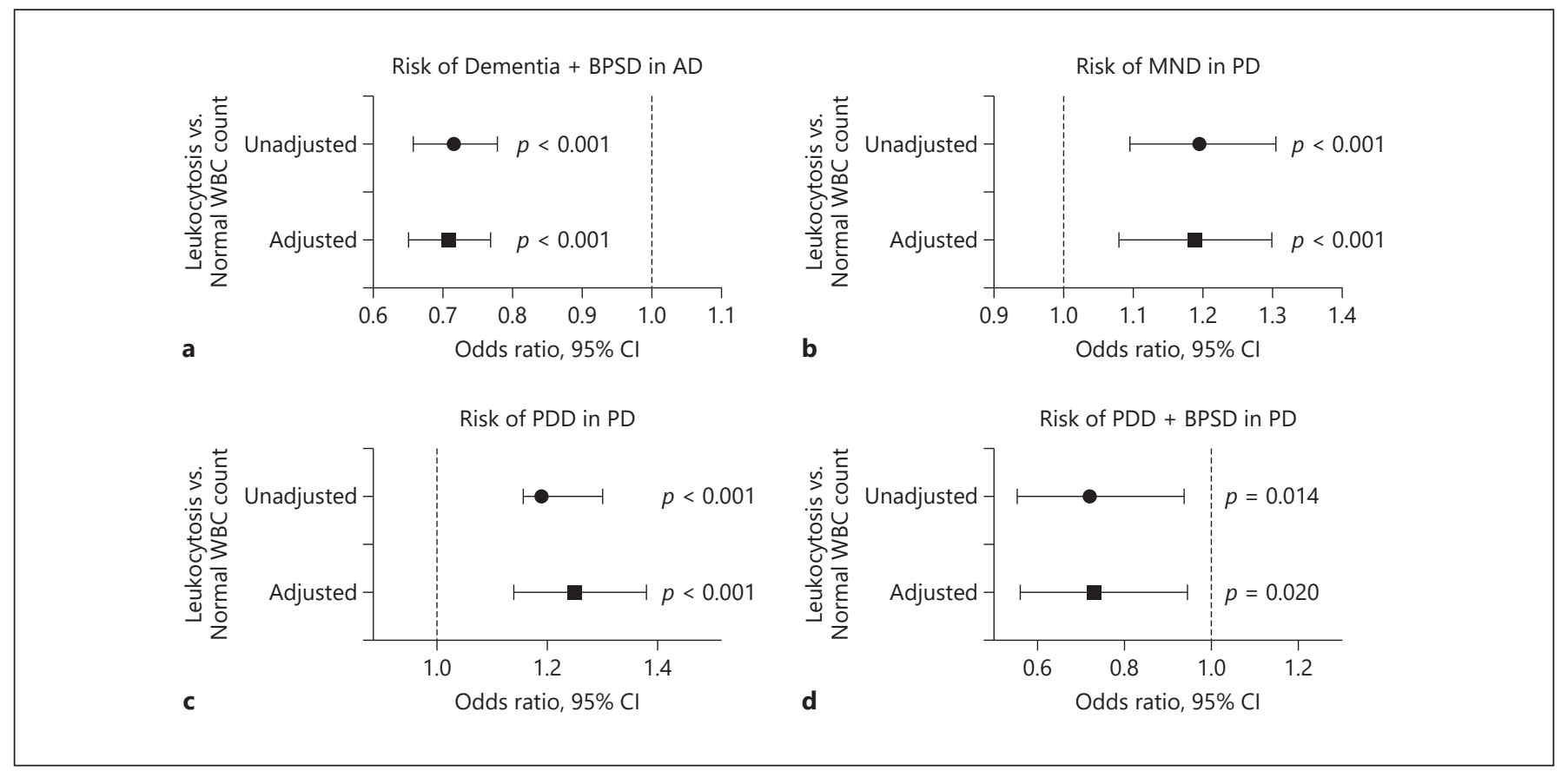

Fig. 3. Leukocytosis is associated with higher odds of cognitive impairment and lower odds of BPSD in AD and PD patients with dementia. a Association of leukocytosis with risk of dementia + BPSD in AD patients. b Association of leukocytosis with risk of MND in PD patients. c Association of leukocytosis with risk of dementia in PD patients. $\mathbf{d}$ Association of leukocytosis with risk of PDD and BPSD. Graph values are based on the total number of AD and PD patients registered in the 2016 and 2017 NIS database. Lo-

$n=679$ vs. $3.0 \%, n=2,163, p<0.001$ ) (Fig. 2d). Similarly, the rate of leukocytosis was higher in PD patients with MCI compared to cognitively normal patients, but this did not reach statistical significance $(3.4 \%, n=2,163$ vs. $3.0 \%, n=24, p=0.592$ ) (Fig. $2 \mathrm{~d}$ ).

A secondary analysis of $\mathrm{PD}$ patients with $\mathrm{MND}$ revealed that, compared to patients with normal cognition, leukocytosis was significantly associated with PDD $(3.8 \%, n=$ 621 vs. $3.0 \%, n=2,163, p<0.001)$, whereas patients with PDD plus BPSD had significantly lower rates of leukocytosis $(2.3 \%, n=58$ vs. $3.0 \%, n=2,163, p=0.039)$ (Fig. 2 f).

Next, we carried out a univariate analysis to assess the unevenly distributed variables in patients with normal and elevated leukocytosis (Table 1). Compared to AD patients with normal WBC count, Alzheimer's patients with leukocytosis were significantly represented by females, Hispanics, hypertensives, current smokers, and individuals with ulcerative colitis $(p<0.05)$. Significantly fewer $\mathrm{AD}$ patients with leukocytosis suffered from complicated diabetes mellitus, congestive heart failure, cerebrovascu- gistic regression analyses show OR, and 95\% CI, for unadjusted (crude) and adjusted (for demographics and comorbidities) leukocytosis contribution to neurocognitive features in $\mathrm{AD}$ and $\mathrm{PD} . \mathrm{AD}$, Alzheimer's disease; PD, Parkinson's disease; WBCs, white blood cells; MND, major neurocognitive disorder; PDD, Parkinson's disease dementia; BPSD, behavioral and psychological symptoms of dementia; NIS, National Inpatient Sample; OR, odds ratio; CI, confidence interval.

lar diseases, sleep apnea, and vascular dementia (Table 1). In the PD cohort, patients with leukocytosis were significantly $(p<0.05)$ represented by whites, hypertensives, diabetics without complications, and current smokers compared to PD patients without leukocytosis. Moreover, PD patients with leukocytosis had significant lower rates of diabetes with complications, congestive heart failure, cerebrovascular diseases, sleep apnea, and asthma (Table 1). All mentioned variables were included to adjust the multiple logistic regression models.

The crude analysis showed a reduced risk of BPSD in $\mathrm{AD}$ disease patients with leukocytosis ( $\mathrm{OR}=0.716,95 \%$ CI: $0.658-0.778, p<0.001)$. After adjusting for variables with $p<0.1$ in the univariate analysis, the multiple logistic regression model (Fig. 3a) showed that leukocytosis was still highly significantly associated with decreased odds of developing BPSD (OR $=0.708$, 95\% CI: 0.651$0.769, p<0.001$ ).

In the PD cohort, WBC count had a crude significant association with an increased risk of MND (OR $=1.19$, 
95\% CI: 1.09-1.31, $p<0.001$ ) (Fig. 3b) but not with MCI $(\mathrm{OR}=1.12$, 95\% CI: $0.743-1.68, p=0.592)$ compared to PD patients without cognitive disturbances (online suppl. Fig. S1). The multiple logistic regression confirmed that leukocytosis was not significantly associated with MCI (OR = 1.14, 95\% CI: 0.777-1.68, $p=0.497)$; however, it was independently associated with higher odds of developing major cognitive disorders $(\mathrm{OR}=1.19,95 \% \mathrm{CI}$ : $1.08-1.30, p<0.001$ ) (online suppl. Fig. S1; Fig. 3b). Similarly, the multiple logistic regression confirmed a significant increased risk for PDD without BPSD in patients with leukocytosis $(\mathrm{OR}=1.25,95 \% \mathrm{CI}: 1.14-1.38, p<$ 0.001 ) (Fig. 3c), and a significant lower risk for patients with PDD and associated BPSD (OR $=0.730,95 \% \mathrm{CI}$ : 0.561-0.95, $p=0.020$ ) (Fig. 3d).

Finally, we performed a matched propensity analysis for both $\mathrm{AD}$ and PD cohorts. The AD cohort included 179,102 age-matched healthy controls out of which 43,617 were excluded due to a diagnosis of infection or fever. A total of 135,485 controls were included in the main analysis. After assessing the distribution of uneven variables between $\mathrm{AD}$ and controls through a univariate analysis (online suppl. Table S7), we proceeded with a multiple logistic regression which revealed a significant association of leukocytosis with risk of $\mathrm{AD}(\mathrm{OR}=1.458,95 \% \mathrm{CI}$ : $1.390-1.529, p<0.001$ ) (online suppl. Table S8). We carried out the same analysis in the PD cohort. A total of 134,501 healthy age-matched controls were included, and after excluding patients with infections or fever, 102,269 controls were considered for the main analysis. Variables with a $p<0.1$ in the univariate analysis (online suppl. Table S7) were included in the adjusted logistic regression, which showed a significant association of leukocytosis with risk of $\mathrm{PD}[\mathrm{OR}=1.187,95 \% \mathrm{CI}: 1.121-1.257$, $p<0.001$ ] (online suppl. Table S9).

\section{Discussion}

With the move toward development of disease-modifying treatments, the identification of diagnostic and prognostic biomarkers that allow for an early characterization and accurate clinical follow-up of $\mathrm{AD}$ and $\mathrm{PD}$ patients with dementia is a major clinical objective. Easy-touse blood-based assays able to provide high specificity and sensitivity are likely to play an important role. In this study, we analyzed the association between pro-inflammatory peripheral immune cells parameters found in routine blood tests and the presence of associated clinical features in $\mathrm{AD}$ and $\mathrm{PD}$. We found that leukocytosis is an

Leukocytosis and Neurocognitive Decline in Alzheimer's and Parkinson's Disease independent predictor of MNDs in PD patients. Furthermore, a sub-analysis to distinguish between patients with PDD only and PDD with associated BPSD revealed that leukocytosis represents a risk factor for dementia, but at the same time is significantly associated with lower risk of BPSD. This association was also seen in the AD cohort were patients with leukocytosis had lower odds of developing BPSD compared to patients with cognitive impairment without behavioral symptoms. Finally, we performed a propensity matched score analysis which revealed that leukocytosis is significantly associated with risk of $\mathrm{AD}$ and $\mathrm{PD}$, supporting our hypothesis that peripheral immune cell levels are elevated in $\mathrm{AD}$ and $\mathrm{PD}$ compared to healthy controls.

Systemic inflammatory burden product of multiple infections has been linked to PD [30] and AD onset [31]. The opposite association of leukocytosis with cognitive and noncognitive symptoms that was found in our study could be explained by the following reasons. First, it has been suggested that levels of systemic inflammatory markers are greater during early stages of $\mathrm{PD}$ and $\mathrm{AD}[9$, 32-34], when they can amplify or even trigger chronic CNS neuroinflammation leading to neurodegeneration. Therefore, symptoms appearing at late stages of $\mathrm{AD}$ and $\mathrm{PD}$, such as BPSD, could more significantly correlate with the progressive neurodegeneration rather than with systemic inflammation. In fact, several studies reported that appearance of BPSD symptoms strongly associate with the degree of functional and cognitive impairment $[41,42]$. For instance, behavioral disturbances such as aggressive behavior tend to occur more often in late stages of $\mathrm{AD}$, which are characterized by severe cognitive deficits and often result in impaired verbal communication $[42,43]$. However, a study of the natural history of BPSD in AD reported that behavioral disturbances can occur almost at any time during the course of the dementia [41], suggesting that the different rates of leukocytosis observed in our study in dementia patients with or without BPSD may not be completely attributed to a chronological factor. Future clinical prospective studies are needed to clarify the dynamic change of peripheral immune cells in relation to the course of $\mathrm{AD}$ and $\mathrm{PD}$.

Second, this phenomenon could be related to the specific mechanisms implicated in cognitive and noncognitive impairment. Loss of cognitive functions in the early stage of dementia is highly associated with neuronal death boosted by the crosstalk between peripheral and CNS inflammation $[44,45]$, which includes different mechanisms such as vagal reflex-induced inflammation from 
the periphery to the brain $[46,47]$, blood-brain barrier disruption [13], and direct entry of peripheral immune cells into the brain parenchyma [12]. Decline of noncognitive functions has been related to changes in anatomic structures [48], circadian rhythm dysfunction [49], metabolic changes [50], and imbalance in the production of neurotransmitters [51, 52], but less is known about how much systemic inflammation aggravates noncognitive symptoms. Hence, our results open a debate on whether there is an underlying mechanism that produces a negative feedback in leukocytes, especially in late stages of dementia with BPSD, making leukocytosis values a reliable biomarker to identify CNS inflammation and damage at the time of BPSD onset.

Our work supports previous evidence of leukocytes anomalies with risk of dementia [53], and to our best knowledge, it is the 1st study that reveals this association on a large national scale. We also provide new insights into the role of systemic inflammatory markers found in routine lab tests with regards to BPSD, which may aid future research to address the identification of clinical subtypes, and to develop prevention and management strategies of currently unmet needs in both AD and PD.

Although having a routine laboratory marker, such as leukocyte count, that predicts neurocognitive deterioration would be ideal, the variability and similarities of the complex mechanisms involved in $\mathrm{AD}$ and $\mathrm{PD}$ make a single blood biomarker not sufficient for specific early diagnosis and accurate prognosis of these 2 neurodegenerative diseases. Therefore, our work should be seen in the light of limitations inherent to the nature of a retrospective study. The predictor variable of interest, leukocytosis, is presented in the NIS database as a categorical variable rather than continuous variable and, therefore, it did not allow the identification of a cutoff threshold that could be used to increase the sensitivity and specificity to predict dementia or BPSD. Moreover, an ICD-10th code for preclinical AD is not available. As suggested by the National Institute on Aging and Alzheimer's Association guidelines, identification of preclinical $\mathrm{AD}$ is not meant to be used in the routine clinical diagnostic decision-making [54]. Thus, an association of cognitive decline from preclinical $\mathrm{AD}$ to dementia due to $\mathrm{AD}$ with leukocytosis in this cohort was not possible. Last, for the main outcomes of dementia and BPSD, the degree of severity, time of onset, and BDSP subtype were not available in the NIS data set.

In view of this, our conclusions are not aimed to be generalized for every $\mathrm{PD}$ or $\mathrm{AD}$ case, but rather to overcome limitations of previous relatively small single-cen- ter studies and, to contribute to the clinical evidence of a critical role of systemic inflammation on the progression of the most prevalent neurodegenerative diseases worldwide.

\section{Conclusions}

In our large-scale retrospective study, we found that peripheral leukocytosis is an independent risk factor for PDD, and is significantly associated with reduced risk of developing behavioral and psychological symptoms in both $\mathrm{AD}$ and $\mathrm{PD}$. Although our results need further elucidation on how leukocytes interact with global cognition and BPSD and its predictive value, we believe the communication of this 1 st national study will aid future works in the search of biomarkers that improves physicians' strategies of prevention and management.

\section{Statement of Ethics}

An IRB approval was not required due to the anonymized and retrospective nature of this study.

\section{Conflict of Interest Statement}

The authors have no conflicts of interest to declare.

\section{Funding Sources}

This research and the APC were funded by the National Institute on Aging for R.M. (Grant No. 1R21AG064455-01A1).

\section{Author Contributions}

Conceptualization: S.R.U. and R.M.; methodology: S.R.U.; formal analysis: S.R.U.; resources: A.M.A. and D.J.A.; investigation: S.R.U., A.M.A., and R.M.; data curation: S.R.U.; writing - original draft preparation: S.R.U. and R.M.; writing - review and editing: S.R.U., A.M.A., D.J.A., and R.M.; supervision: R.M.; funding acquisition: R.M.; project administration: R.M. All authors have read and agreed to the published version of the manuscript. 


\section{References}

1 Walker KA. Inflammation and neurodegeneration: chronicity matters. Aging. 2018; 11(11):3-4.

2 Heneka MT, Carson MJ, El Khoury J, Landreth GE, Brosseron F, Feinstein DL, et al. Neuroinflammation in Alzheimer's disease. Lancet Neurol. 2015;14(4):388-405.

3 Jevtic S, Sengar AS, Salter MW, McLaurin J. The role of the immune system in Alzheimer disease: etiology and treatment. Ageing Res Rev. 2017;40(40):84-94.

4 Gelders G, Baekelandt V, Van der Perren A. Linking neuroinflammation and neurodegeneration in Parkinson's disease. J Immunol Res. 2018;2018(2018):4784268.

5 Joshi N, Singh S. Updates on immunity and inflammation in Parkinson disease pathology. J Neurosci Res. 2018;96(96):379-90.

6 Kempuraj D, Thangavel R, Natteru PA, Selvakumar GP, Saeed D, Zahoor H, et al. Neuroinflammation Induces Neurodegeneration. J Neurol Neurosurg Spine. 2016;1(1).

7 Serpente M, Bonsi R, Scarpini E, Galimberti D. Innate immune system and inflammation in Alzheimer's disease: from pathogenesis to treatment. Neuroimmunomodulation. 2014; 21(21):79-87.

8 Johnson ME, Stecher B, Labrie V, Brundin L, Brundin P. Triggers, facilitators, and aggravators: redefining Parkinson's disease pathogenesis. Trends Neurosci. 2019;42(42):4-13.

9 Kim R, Kim HJ, Kim A, Jang M, Kim A, Kim $Y$, et al. Peripheral blood inflammatory markers in early Parkinson's disease. J Clin Neurosci. 2018;58(58):30-3.

10 Qiu X, Xiao Y, Wu J, Gan L, Huang Y, Wang J. C-reactive protein and risk of Parkinson's d: a systematic review and meta-analysis. Front Neurol. 2019;10(10):384

11 Sankowski R, Mader S, Valdes-Ferrer SI. Systemic inflammation and the brain: novel roles of genetic, molecular, and environmental cues as drivers of neurodegeneration. Front Cell Neurosci. 2015;9:28.

12 Ferrari CC, Tarelli R. Parkinson's disease and systemic inflammation. Parkinsons Dis. 2011; 2011(2011):436813

13 Fakhoury M. Role of immunity and inflammation in the pathophysiology of neurodegenerative diseases. Neurodegener Dis. 2015; 15(2):63-9.

14 Poewe W, Seppi K, Tanner CM, Halliday GM, Brundin P, Volkmann J, et al. Parkinson disease. Nat Rev Dis Primers. 2017;3(3):17013.

15 Marras C, Beck JC, Bower JH, Roberts E, Ritz B, Ross GW, et al. Prevalence of Parkinson's disease across North America. NPJ Parkinsons Dis. 2018;4(4):21.

16 Alzheimer's Association. 2016 Alzheimer's disease facts and figures. Alzheimers Dement. 2016;12(4):459-509.
17 Scheltens P, Blennow K, Breteler MM, de Strooper B, Frisoni GB, Salloway S, et al. Alzheimer's disease. Lancet. 2016;388(388):50517.

18 Santos-Garcia D, Fonticoba TD, Castro ES, Diaz AA, Gonzalez JMP, Panceiras MJF, et al. High ultrasensitive serum C-reactive protein may be related to freezing of gait in Parkinson's disease patients. J Neural Transm. 2019; 126(126):1599-608.

19 Tan ZS, Beiser AS, Vasan RS, Roubenoff R, Dinarello CA, Harris TB, et al. Inflammatory markers and the risk of Alzheimer disease: the Framingham Study. Neurology. 2007;68(22): 1902-8.

20 Mollenhauer B, Zimmermann J, Sixel-Doring F, Focke NK, Wicke T, Ebentheuer J, et al. Baseline predictors for progression 4 years after Parkinson's disease diagnosis in the De Novo Parkinson Cohort (DeNoPa). Mov Disord. 2019;34(34):67-77.

21 Buter TC, van den Hout A, Matthews FE, Larsen JP, Brayne C, Aarsland D. Dementia and survival in Parkinson disease: a 12-year population study. Neurology. 2008;70(13): 1017-22.

22 Lindqvist D, Kaufman E, Brundin L, Hall S, Surova Y, Hansson O. Non-motor symptoms in patients with Parkinson's disease: correlations with inflammatory cytokines in serum. PLoS One. 2012;7(7):e47387.

23 Gottesman RT, Stern Y. Behavioral and psychiatric symptoms of dementia and rate of decline in Alzheimer's disease. Front Pharmacol. 2019;10(10):1062.

24 Gauthier S, Cummings J, Ballard C, Brodaty H, Grossberg G, Robert P, et al. Management of behavioral problems in Alzheimer's disease. Int Psychogeriatr. 2010;22(3):346-72.

25 Aigbogun MS, Stellhorn R, Hartry A, Baker RA, Fillit H. Treatment patterns and burden of behavioral disturbances in patients with dementia in the United States: a claims database analysis. BMC Neurol. 2019;19(19):33.

26 Chiu PY, Tsai CT, Chen PK, Chen WJ, Lai TJ. Neuropsychiatric symptoms in Parkinson's disease dementia are more similar to Alzheimer's disease than dementia with Lewy bodies: a case-control study. PLoS One. 2016; 11(4):e0153989.

27 Goldman JG, Vernaleo BA, Camicioli R, Dahodwala N, Dobkin RD, Ellis T, et al. Cognitive impairment in Parkinson's disease: a report from a multidisciplinary symposium on unmet needs and future directions to maintain cognitive health. NPJ Parkinsons Dis. 2018;4(4):19.

28 Aarsland D, Andersen K, Larsen JP, Lolk A, Nielsen H, Kragh-Sorensen P. Risk of dementia in Parkinson's disease: a communitybased, prospective study. Neurology. 2001; 56(6):730-6.
29 Lin CH, Chiu SI, Chen TF, Jang JSR, Chiu MJ. Classifications of neurodegenerative disorders using a multiplex blood biomarkersbased machine learning model. Int J Mol Sci. 2020;21(18):6914.

30 Bu XL, Wang X, Xiang Y, Shen LL, Wang QH, Liu $\mathrm{YH}$, et al. The association between infectious burden and Parkinson's disease: a casecontrol study. Parkinsonism Relat Disord. 2015;21(8):877-81.

31 Bu XL, Yao XQ, Jiao SS, Zeng F, Liu YH, Xiang Y, et al. A study on the association between infectious burden and Alzheimer's disease. Eur J Neurol. 2015;22(22):1519-25.

32 Herlofson K, Heijnen CJ, Lange J, Alves G, Tysnes OB, Friedman JH, et al. Inflammation and fatigue in early, untreated Parkinson's disease. Acta Neurol Scand. 2018;138(138): 394-9.

33 Park JC, Han SH, Mook-Jung I. Peripheral inflammatory biomarkers in Alzheimer's disease: a brief review. BMB Rep. 2020;53(53): 10-9.

34 Williams-Gray CH, Wijeyekoon R, Yarnall AJ, Lawson RA, Breen DP, Evans JR, et al. Serum immune markers and disease progression in an incident Parkinson's disease cohort (ICICLE-PD). Mov Disord. 2016;31(7):9951003.

35 Albert MS, DeKosky ST, Dickson D, Dubois B, Feldman HH, Fox NC, et al. The diagnosis of mild cognitive impairment due to $\mathrm{Alz}$ heimer's disease: recommendations from the National Institute on Aging-Alzheimer's Association workgroups on diagnostic guidelines for Alzheimer's disease. Alzheimers Dement. 2011;7(7):270-9.

36 McKhann GM, Knopman DS, Chertkow H, Hyman BT, Jack CR, Kawas CH, et al. The diagnosis of dementia due to Alzheimer's disease: recommendations from the National Institute on Aging-Alzheimer's Association workgroups on diagnostic guidelines for Alzheimer's disease. Alzheimers Dement. 2011; 7(3):263-9.

37 American Psychiatric Association. Diagnostic and statistical manual of mental disorders. 5th ed. Arlington: American Psychiatric Publishing; 2013.

38 Cloak N, Al Khalili Y. Behavioral and psychological symptoms in dementia [Internet]. Treasure Island: StatPearls Publishing; 2020.

39 Kales HC, Kern V, Kim HM, Blazek MC. Moving evidence-informed assessment and management of behavioral and psychological symptoms of dementia into the real world: training family and staff caregivers in the DICE approach. Am J Geriatr Psychiatry. 2020;28(28):1248-55.

40 Mank V, Brown K. Leukocytosis [Internet]. Treasure Island, FL: StatPearls Publishing; 2020 . 
41 Hope T, Keene J, Fairburn CG, Jacoby R, McShane R. Natural history of behavioural changes and psychiatric symptoms in Alzheimer's disease. A longitudinal study. Br J Psychiatry. 1999;174:39-44.

42 Yi SH, Suk SH. Letter to the editor: relationship between neuropsychiatric symptoms and activities of daily living in Alzheimer disease. Ann Geriatr Med Res. 2018;22(22):4950.

43 Finkel SI, Costa e Silva J, Cohen G, Miller S, Sartorius N. Behavioral and psychological signs and symptoms of dementia: a consensus statement on current knowledge and implications for research and treatment. Int Psychogeriatr. 1996;8(Suppl 3):497-500.

44 Paouri E, Gcorgopoulos S. Systemic and CNS inflammation crosstalk: implications for $\mathrm{Alz}$ heimer's Disease. Curr Alzheimer Res. 2019; 16(16):559-74.

45 Wallings RL, Herrick MK, Tansey MG. LRRK2 at the interface between peripheral and central immune function in Parkinson's. Front Neurosci. 2020;14(14):443.
46 Perry VH, Cunningham C, Holmes C. Systemic infections and inflammation affect chronic neurodegeneration. Nat Rev Immunol. 2007;7(2):161-7.

47 Tracey KJ. The inflammatory reflex. Nature. 2002 Dec;420(6917):853-9.

48 Kwak S, Park S, Kim J, Park S, Lee JY. Multivariate neuroanatomical correlates of behavioral and psychological symptoms in dementia and the moderating role of education. Neuroimage Clin. 2020;28(28):102452.

49 Todd WD. Potential pathways for circadian dysfunction and sundowning-related behavioral aggression in Alzheimer's disease and related dementias. Front Neurosci. 2020; 14(14):910.

50 Ishii M, Iadecola C. Metabolic and non-cognitive manifestations of Alzheimer's disease: the hypothalamus as both culprit and target of pathology. Cell Metab. 2015;22(5):761-76.
51 Angelucci F, Bernardini S, Gravina P, Bellincampi L, Trequattrini A, Di Iulio F, et al. Delusion symptoms and response to antipsychotic treatment are associated with the 5-HT2A receptor polymorphism (102T/C) in Alzheimer's disease: a 3-year follow-up longitudinal study. J Alzheimers Dis. 2009;17(17):203-11.

52 Proitsi P, Lupton MK, Reeves SJ, Hamilton G, Archer N, Martin BM, et al. Association of serotonin and dopamine gene pathways with behavioral subphenotypes in dementia. Neurobiol Aging. 2012;33(4):791-803.

53 Martin-Ruiz C, Williams-Gray CH, Yarnall AJ, Boucher JJ, Lawson RA, Wijeyekoon RS, et al. Senescence and inflammatory markers for predicting clinical progression in Parkinson's disease: the ICICLE-PD study. J Parkinsons Dis. 2020;10(10):193-206.

54 McKhann GM, Knopman DS, Chertkow H, Hyman BT, Jack CR Jr, Kawas CH, et al. The diagnosis of dementia due to Alzheimer's disease: recommendations from the National Institute on Aging-Alzheimer's Association workgroups on diagnostic guidelines for Alzheimer's disease. Alzheimers Dement. 2011; $7(3): 263-9$. 